\title{
COMPARISION OF PROPORTION AND RESISTANCE RATIO METHODS FOR DRUG SUSCEPTIBILITY TESTING OF Mycobacterium tuberculosis ISOLATED FROM PATIENTS ATTENDING NATIONAL TUBERCULOSIS CENTRE, NEPAL
}

\author{
Acharya $\mathbf{S}^{1}$, Ghimire $\mathbf{P}^{1}$, Khadka D. K. ${ }^{2}$, Nepali S. ${ }^{1}$ \\ ${ }^{1}$ Central Department of Microbiology, Tribhuvan University, Kirtipur, Kathmandu, Nepal \\ 2 SAARC Tuberculosis and HIVIAIDS Centre, Thimi, Bhaktapur, Nepal
}

\begin{abstract}
Background: Tuberculosis (TB) is among the most serious infectious cause of global morbidity and mortality. Emergence of Multi-drug resistant tuberculosis (MDR-TB) is posing an increased threat to TB control programs. Drug susceptibility testing (DST) of Mycobacterium tuberculosis (M. tuberculosis) isolates is important for tackling such problems.

Setting: National Tuberculosis Centre (NTC), Thimi, Bhaktapur, Nepal.

Objectives: Comparative evaluation of two in vitro DST methods in determining susceptibility of $M$. tuberculosis isolates from patients attending NTC, to front-line anti-TB drugs: (Isoniazid-INH, Rifampicin-RFP, Streptomycin-SM, and Ethambutol-EMB).

Methodology: This study was conducted from Sep 2006-Jun 2007. A total of 862 sputum samples (diagnosis or follow up cases) collected from patients (type of patients or their categories was not differentiated in this study) attending NTC bacteriology lab for sputum direct smear microscopy were analyzed using fluorescence microscopy. All smear positive samples, smear negative samples requested for culture were cultured. All culture positive samples confirmed as M. tuberculosis by biochemical tests were processed for DST by both proportion (PR) and resistance ratio (RR) methods. Results: Out of 862 sputum samples analyzed, 226 (26.2\%) samples were positive for Acid Fast Bacilli (AFB) by fluorescence microscopy. Among 323 samples 226 smear positive samples and 97 smear negative samples requested for culture), $221(68.4 \%)$ were culture positive, $92(28.5 \%)$ were culture negative and $10(3.1 \%)$ were contaminated. Out of 221 isolates of $M$. tuberculosis, $57.5 \%$ were resistant to one or more drugs by the PR method and $56.6 \%$ by the RR method. Similarly, MDR isolates were $29.9 \%$ and $29 \%$ by PR and RR methods respectively.

On correlation analysis using Mc Nemar Chi-square test, no significant difference between the two tests were observed ( $p>0.05)$. The results showed high agreement between both methods and agreement rates to INH, RFP, SM and EMB were $93.2 \%, 93.7 \%, 93.2 \%$ and $94.1 \%$ respectively. Similarly, the agreement rates between both methods using kappa analysis showed kappa $(\mathrm{k})$ value of $0.86,0.85$, 0.86 and 0.84 for INH, RFP, SM and EMB respectively, which is believed to be good agreement between both methods ( $k=0.80$ to 1.00: Very good agreement).

Conclusion: In conclusion, this study showed that both the Proportion and Resistance ratio methods are equally good for determining drug susceptibility of $M$. tuberculosis.

Keywords: Mycobacterium tuberculosis, Drug Susceptibility Testing, Proportion Method, Resistance Ratio Method.

Correspondence to:

Sushma Acharya

C/O: Dr. Prakash Ghimire

Central Department of Microbiology, Tribhuvan University, Kirtipur,

Kathmandu, Nepal.

E-mail: sushma_18@hotmail.com prakashghimire@yahoo.com
\end{abstract}




\section{Introduction}

Tuberculosis (TB) constitutes a major public health problem in most developing countries of the world accounting for the largest burden of mortality due to any infectious agent worldwide. It is estimated that up to one-third of the world's population is infected with the tubercle bacilli along with the emergence of 450,000 new multidrug resistant TB (MDR-TB) cases every year. ${ }^{1}$ In Nepal, about $45 \%$ of the total population is infected with TB, of which $60 \%$ are adult. Every year, 40,000 people develop active TB, of whom 20,000 have infectious pulmonary disease and 5,000 to 7,000 people die from TB. ${ }^{2}$ Co-infection with the human immunodeficiency virus (HIV) significantly increases the risk of developing TB. ${ }^{3}$

According to $\mathrm{WHO}$, more people will die of TB this year than in any other year in history. Of equal concern, however is the emergence and transmission of MDR strains of $M$. tuberculosis. In light of this frightening scenario, laboratory strategies for reliable drug susceptibility testing (DST) of M. tuberculosis is of prime importance.

There are different conventional methods (proportion, resistance ratio, absolute concentration methods), radiometric method and other newer methods used for determining antimicrobial susceptibility pattern. The most extensively used being the proportion (PR) and the resistance ratio (RR) methods. The PR method compares the degree of growth of test organism in drug containing media and drug free media; whereas in the RR method, the resistance of unknown strain of tubercle bacilli (test organism) is compared with that of a standard laboratory strain of $M$. tuberculosis $\left(\mathrm{H}_{37} \mathrm{Rv}\right) .{ }^{4}$

The accurate DST method is very important to determine the exact susceptibility pattern of $M$. tuberculosis and hence this study is undertaken to compare the two methods (the PR and the RR methods) for susceptibility testing of $M$. tuberculosis and to find out the agreement rate between them.

\section{Methodology}

The study was based at National Tuberculosis Centre (NTC), Thimi, Bhaktapur, Nepal.
This study was carried out from Sep 2006-Jun 2007. A total of 862 sputum samples, collected from patients attending NTC, were analyzed further.

\section{Laboratory methodology}

a) Specimen collection, microscopic examination and culture: The sputum samples were collected from patients at NTC as per the standard protocol. ${ }^{5}$ All the sputum specimens were processed for direct smear examination by fluorescence microscopy. ${ }^{5}$ Positive slides were further confirmed by Ziehl-Neelsen staining. Smear positive samples and culture requested smear negative samples were cultured on $2 \%$ Modified Ogawa medium. Mycobacterial cultures were incubated at $37^{\circ} \mathrm{C}$ for 8 weeks with weekly observation for growth. Bacterial colonies were identified as $M$. tuberculosis by colony characteristics and biochemical tests. 5,6

b) Drug susceptibility test: All biochemically confirmed $M$. tuberculosis were subjected for susceptibility studies. The susceptibilities of these strains to each four primary anti-tubercular drugs were tested by both PR and RR methods.

i. Proportion Method: Tests were performed using a standard variant of the proportion

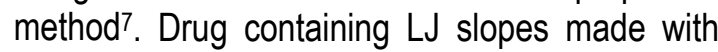
the critical drug concentrations for INH, RFP, SM and EMB were $0.25,40,4$ and $2 \mu \mathrm{g} / \mathrm{ml}$, respectively. The drug free control media were prepared at the same time. The standardized 1 $\mathrm{mg} / \mathrm{ml}$ bacillary suspension ${ }^{6}$ (McFarland No. 1) was diluted in sterile distilled water to give $10^{-1}$, $10^{-2}$ and $10^{-3}$ dilutions. From $10^{-1}$ dilution, all drug containing media were inoculated with one loopfull of bacillary suspension. Similarly, from each dilution, three controls of plain LJ media were inoculated with the respective diluted bacillary suspension. The slopes were incubated at $37^{\circ} \mathrm{C}$, and the results were read on the $28^{\text {th }}$ day. Any colonies growing on drug containing medium inoculated with the $10^{-1}$ dilution that equal or more the number of colonies growing on the control medium inoculated with the $10^{-3}$ dilution represents $1 \%$ or more of the test population. If the calculation was $1 \%$ or more then interpreted resistance. 
ii. Resistance Ratio method: Parallel sets of media containing two-fold dilutions of the primary anti-TB drugs were prepared as follows: $\mathrm{INH}, 0.5$ and $1.0 \mu \mathrm{g} / \mathrm{ml}$; RFP, 32.0 and $64.0 \mu \mathrm{g} / \mathrm{ml} ; \mathrm{SM}$, 16.0 and $32.0 \mu \mathrm{g} / \mathrm{ml}$; and $\mathrm{EMB}, 4.0$ and 8.0 $\mu \mathrm{g} / \mathrm{ml} .8$ One drop $(100 \mu \mathrm{l})$ of $1 \mathrm{mg} / \mathrm{ml}$ bacillary suspension (McFarland No. 1) from a Pasteur pipette was spread on the surface of each eight drug containing slopes of media of different concentrations. Similar procedure was applied for $\mathrm{H}_{37} \mathrm{Rv}$ strain. All these tubes were incubated at $37^{\circ} \mathrm{C}$ for 4 weeks and observed weekly. For all tests, growth was defined as the presence of 20 or more colonies in the drug containing media. The isolate was considered resistant when the growth appeared on the media containing a given drug in a given concentration in which control strain is susceptible.

Data analysis: All the collected data was processed and analyzed using MS-Excel 2003 and SPSS version 11.5.

\section{Results}

AFB smear microscopy and culture: Out of 862 samples, $588(68.2 \%)$ were from male and $274(31.8 \%)$ were from female. Out of 862 sputum samples, 226 (26.2\%) showed AFB on fluorescence staining; of which $173(76.5 \%)$ were from male and $53(23.5 \%)$ were from female (Table 1 and 2).

Out of 323 samples (226 smear positive samples and 97 smear negative samples requested for culture), $221(68.4 \%)$ samples were culture positive, $92(28.5 \%)$ samples were culture negative and $10(3.1 \%)$ samples were contaminated (Table 3).

Drug susceptibility studies: All 221 biochemically confirmed $M$. tuberculosis were subjected for susceptibility studies. The susceptibilities of these strains to each drug tested by the PR and RR methods are listed in Table 4. The results of both methods were compared for the rates of susceptible and resistance of strains to all 4 drugs (Table 5). For the PR method; 94 of 221 strains (42.5\%) were susceptible to all four drugs, and 127 of 221 $(57.5 \%)$ were resistant to at least one drug. For the RR method; 96 of 221 strains (43.4\%) were susceptible to all four drugs, and 125 of 221 $(56.6 \%)$ were resistant to at least one drug. The results of susceptible and resistant rates of $M$. tuberculosis to these drugs determined by both methods were in very good agreement $(k=0.852) \cdot{ }^{9}$

As shown in Table 6, the PR method identified $57.5 \%$ of the isolates as resistant to at least one of the four drugs. Resistance to INH and SM was the highest as both of them at $5.4 \%$, while resistance to RFP was $0.5 \%$ and there was no EMB only resistant strain. Resistance to one, two, three and four drugs was observed in 11.3, $14.9,14.0$, and $17.1 \%$ of the isolates respectively. MDR was found in $29.9 \%$ of the isolates. Resistance to SM and others, INH and others, RFP and others, and EMB and others was found in 44.8, 50.2, 31.2, and $25.8 \%$ respectively.

The RR method identified $56.6 \%$ of the isolates as resistant strains. Resistance to INH was the highest at $5.9 \%$, while resistance to RFP and SM were 1.8 and $4.5 \%$ respectively. There were no EMB only resistant strains. Resistance to one, two, three and four drugs was observed in 12.2, $17.7,13.6$, and $13.1 \%$ respectively. MDR was found in $29 \%$ of the isolates. Resistance to SM and others, INH and others, RFP and others, and EMB and others was found in 39.82, 47.06, 32.13 , and $21.72 \%$ respectively.

Agreement between Proportion and Resistance ratio methods: The percentages of agreement between the PR and the RR methods for antimicrobial susceptibility of $221 \mathrm{M}$. tuberculosis to INH, RFP, SM and EMB were 93.2, 93.7, 93.2 and $94.1 \%$, respectively (Table 7).

Correlation between both methods for determining susceptibilities of these strains to four drugs tested is shown in Table 8. There was high agreement between both methods when tested against INH, RFP, SM and EMB with kappa, $\mathrm{k}=0.86,0.85,0.86$ and 0.84 respectively. Statistical comparison using Mc Nemar $X^{2}$ test showed the value $0.118,0.791,0.007$ and 0.022 for INH, RFP, SM and EMB respectively. The tabulated value of chi square at $5 \%$ level of 
significance for 1 degree of freedom is $3.84 .^{18}$ Thus calculated chi square value was less than tabulated value which revealed that there was no statistically significant difference between both methods for determining susceptibilities to all the four drugs tested $(p>0.05)$.

Table 1 Age and gender-wise distribution of patients attending NTC

\begin{tabular}{|c|c|c|c|c|c|c|c|}
\hline \multirow{3}{*}{$\begin{array}{l}\text { S. } \\
\text { No. }\end{array}$} & \multirow{3}{*}{ Age group (years) } & \multicolumn{6}{|c|}{ Gender of the patients } \\
\hline & & \multicolumn{2}{|c|}{ Male } & \multicolumn{2}{|c|}{ Female } & \multicolumn{2}{|c|}{ Total } \\
\hline & & Count & $\%$ of total & Count & $\%$ of total & Count & $\%$ of total \\
\hline 1 & $5-10$ yrs & 2 & $0.2 \%$ & 1 & $0.1 \%$ & 3 & $0.3 \%$ \\
\hline 2 & $11-15$ yrs & 12 & $1.4 \%$ & 14 & $1.6 \%$ & 26 & $3.0 \%$ \\
\hline 3 & $16-20$ yrs & 54 & $6.3 \%$ & 29 & $3.4 \%$ & 83 & $9.6 \%$ \\
\hline 4 & $21-25$ yrs & 77 & $8.9 \%$ & 57 & $6.6 \%$ & 134 & $15.5 \%$ \\
\hline 5 & $26-30$ yrs & 80 & $9.3 \%$ & 36 & $4.2 \%$ & 116 & $13.5 \%$ \\
\hline 6 & $31-35$ yrs & 74 & $8.6 \%$ & 17 & $2.0 \%$ & 91 & $10.6 \%$ \\
\hline 7 & $36-40$ yrs & 43 & $5.0 \%$ & 28 & $3.2 \%$ & 71 & $8.2 \%$ \\
\hline 8 & $41-45$ yrs & 57 & $6.6 \%$ & 21 & $2.4 \%$ & 78 & $9.0 \%$ \\
\hline 9 & $46-50$ yrs & 55 & $6.4 \%$ & 26 & $3.0 \%$ & 81 & $9.4 \%$ \\
\hline 10 & $51-55$ yrs & 30 & $3.5 \%$ & 14 & $1.6 \%$ & 44 & $5.1 \%$ \\
\hline 11 & $56-60$ yrs & 34 & $3.9 \%$ & 14 & $1.6 \%$ & 48 & $5.6 \%$ \\
\hline 12 & Above 60 yrs & 70 & $8.1 \%$ & 17 & $2.0 \%$ & 87 & $10.1 \%$ \\
\hline Total & & 588 & $68.2 \%$ & 274 & $31.8 \%$ & 862 & $100 \%$ \\
\hline
\end{tabular}

Table 2 Gender-wise distribution of fluorochrome stain of the samples

\begin{tabular}{|c|c|c|c|c|c|c|c|}
\hline \multirow{3}{*}{ S. No. } & \multirow{3}{*}{$\begin{array}{c}\text { Fluorescence } \\
\text { staining }\end{array}$} & \multicolumn{6}{|c|}{ Gender of the patients } \\
\hline & & \multicolumn{2}{|c|}{ Male } & \multicolumn{2}{|c|}{ Female } & \multicolumn{2}{|c|}{ Total } \\
\hline & & Count & $\%$ of total & Count & $\%$ of total & Count & $\%$ of total \\
\hline 1 & Negative & 415 & $48.1 \%$ & 221 & $25.6 \%$ & 636 & $73.8 \%$ \\
\hline 2 & $1+$ & 84 & $9.7 \%$ & 25 & $2.9 \%$ & 109 & $12.6 \%$ \\
\hline 3 & $2+$ & 53 & $6.1 \%$ & 14 & $1.6 \%$ & 67 & $7.8 \%$ \\
\hline 4 & $3+$ & 36 & $4.2 \%$ & 14 & $1.6 \%$ & 50 & $5.8 \%$ \\
\hline Total & & 588 & $68.2 \%$ & 274 & $31.8 \%$ & 862 & $100 \%$ \\
\hline
\end{tabular}

Table 3 Gender-wise distribution of culture results

\begin{tabular}{|c|c|c|c|c|}
\hline \multirow{3}{*}{ S. No. } & \multirow{2}{*}{ Culture results } & \multicolumn{3}{|c|}{ Gender of the patients } \\
\cline { 3 - 5 } & & Male & Female & Total \\
\cline { 3 - 5 } & $1+$ & Count & Count & Count \\
\hline 1 & $2+$ & 39 & 15 & 54 \\
\hline 2 & $3+$ & 43 & 13 & 56 \\
\hline 3 & $4+$ & 85 & 16 & 101 \\
\hline 4 & 6 & 4 & 10 \\
\hline 5 & Contamination & 8 & 2 & 10 \\
\hline 6 & Negative & 64 & 28 & 92 \\
\hline Total & & $\mathbf{2 4 5}$ & $\mathbf{7 8}$ & $\mathbf{3 2 3}$ \\
\hline
\end{tabular}

Table 4 Pattern of susceptibilities to four anti-tuberculosis drugs determined by Proportion and Resistance ratio methods

\begin{tabular}{|c|c|c|c|c|c|}
\hline \multirow{2}{*}{ S. No. } & \multirow{2}{*}{ Drugs } & \multicolumn{2}{|c|}{ Proportion method } & \multicolumn{2}{c|}{ Resistance ratio method } \\
\cline { 3 - 6 } & & Susceptible & Resistant & Susceptible & Resistant \\
\hline 1 & INH & 110 & 111 & 117 & 104 \\
\hline 2 & RFP & 152 & 69 & 150 & 81 \\
\hline 3 & SM & 122 & 99 & 133 & 48 \\
\hline 4 & EMB & 164 & 57 & 173 & \\
\hline
\end{tabular}


Table 5 Comparison between the Proportion and the Resistance ratio methods for susceptible and resistance of isolates of $M$. tuberculosis to all four primary anti-tubercular drugs

\begin{tabular}{|l|c|c|c|}
\hline \multirow{2}{*}{ RR method } & \multicolumn{2}{|c|}{ PR method } & \multirow{2}{*}{ Total } \\
\cline { 2 - 3 } & Susceptible & Resistant & 96 \\
\hline Susceptible & 87 & 9 & 125 \\
\hline Resistant & 7 & 118 & 221 \\
\hline Total & 94 & 127 & \\
\hline
\end{tabular}

$K=0.852$

Table 6 Patterns of resistance of $M$. tuberculosis to primary anti-tuberculosis drugs determined by the Proportion and the Resistance Ratio methods

\begin{tabular}{|c|c|c|c|c|}
\hline \multirow{2}{*}{ Pattern } & \multicolumn{4}{|c|}{ No. of strains } \\
\hline & PR method & $\%$ & RR method & $\%$ \\
\hline Resistance & 127 & 57.5 & 125 & 56.56 \\
\hline Mono-resistance to & 25 & 11.3 & 27 & 12.2 \\
\hline \multirow{4}{*}{$\begin{array}{l}\text { INH } \\
\text { RFP } \\
\text { SM } \\
\text { EMB }\end{array}$} & 12 & 5.4 & 13 & 5.9 \\
\hline & 1 & 0.5 & 4 & 1.8 \\
\hline & 12 & 5.4 & 10 & 4.5 \\
\hline & 0 & -- & 0 & -- \\
\hline \multirow{7}{*}{$\begin{array}{l}\text { Resistance to } 2 \text { drugs } \\
\qquad \begin{array}{r}\text { SM+INH } \\
\text { SM+RFP } \\
\text { SM+EMB } \\
\text { INH+RFP } \\
\text { INH+EMB } \\
\text { RFP+EMB }\end{array}\end{array}$} & 33 & 14.9 & 39 & 17.7 \\
\hline & 25 & 11.3 & 21 & 9.5 \\
\hline & 0 & -- & 1 & 0.5 \\
\hline & 1 & 0.5 & 4 & 1.8 \\
\hline & 3 & 1.4 & 10 & 4.5 \\
\hline & 3 & 1.4 & 2 & 0.9 \\
\hline & 1 & 0.5 & 1 & 0.5 \\
\hline \multirow{5}{*}{ 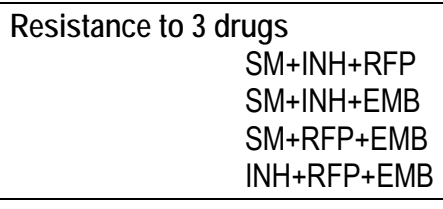 } & 31 & 14.0 & 30 & 13.6 \\
\hline & 17 & 7.7 & 18 & 8.1 \\
\hline & 5 & 2.3 & 4 & 1.8 \\
\hline & 1 & 0.5 & 1 & 0.5 \\
\hline & 8 & 3.6 & 7 & 3.2 \\
\hline $\begin{array}{l}\text { Resistance to } 4 \text { drugs } \\
\qquad \mathrm{SM}+\mathrm{INH}+\mathrm{RFP}+\mathrm{EMB}\end{array}$ & 38 & 17.2 & 29 & 13.1 \\
\hline \multirow{5}{*}{$\begin{array}{l}\text { MDR-TB } \\
\text { INH+RFP } \\
\text { SM+INH+RFP } \\
\text { INH+RFP+EMB } \\
\text { SM+INH+RFP+EMB }\end{array}$} & 66 & 29.9 & 64 & 29.0 \\
\hline & 3 & 1.4 & 10 & 4.5 \\
\hline & 17 & 7.7 & 18 & 8.1 \\
\hline & 8 & 3.6 & 7 & 3.2 \\
\hline & 38 & 17.2 & 29 & 13.1 \\
\hline Resistance to SM and others & 99 & 44.8 & 88 & 39.8 \\
\hline Resistance to INH and others & 111 & 50.2 & 104 & 47.1 \\
\hline \multirow{2}{*}{ Resistance to RFP and others } & 69 & 31.2 & 71 & 32.1 \\
\hline & 57 & 25.8 & 48 & 21.7 \\
\hline
\end{tabular}

Table 7 Percentage agreements between the Proportion and the Resistance Ratio methods for susceptibility testing of $M$. tuberculosis to each drug tested

\begin{tabular}{|c|c|c|c|c|}
\hline S. No & \multirow{2}{*}{ Drugs } & \multicolumn{2}{|c|}{ No. of isolates with the following results } & Percent agreement \\
\cline { 3 - 5 } & & $\begin{array}{l}\text { PR method-Susceptible } \\
\text { RR method-Susceptible }\end{array}$ & $\begin{array}{l}\text { PR method-Resistant } \\
\text { RR method-Resistant }\end{array}$ & \\
\hline 1 & INH & 106 & 100 & 93.2 \\
\hline 2 & RFP & 144 & 63 & 93.7 \\
\hline 3 & SM & 120 & 86 & 93.2 \\
\hline 4 & EMB & 162 & 46 & 94.1 \\
\hline
\end{tabular}


Table 8 Comparison between the Proportion and the Resistance Ratio methods for determining susceptibility of $M$. tuberculosis to four primary anti-tubercular drugs

\begin{tabular}{|c|c|c|c|c|c|c|}
\hline \multirow{2}{*}{ S. No. } & \multirow{2}{*}{ Drugs } & \multirow{2}{*}{ RR method } & \multicolumn{2}{|c|}{ PR method } & \multirow{2}{*}{ Total } & \multirow{2}{*}{$\mathrm{K}$} \\
\hline & & & Susceptible & Resistant & & \\
\hline \multirow{3}{*}{1} & \multirow{3}{*}{ INH } & Susceptible & 106 & 11 & 117 & \multirow{3}{*}{0.86} \\
\hline & & Resistant & 4 & 100 & 104 & \\
\hline & & Total & 110 & 111 & 221 & \\
\hline \multirow{3}{*}{2} & \multirow{3}{*}{ RFP } & Susceptible & 144 & 6 & 150 & \multirow{3}{*}{0.8} \\
\hline & & Resistant & 8 & 63 & 71 & \\
\hline & & Total & 152 & 69 & 221 & \\
\hline \multirow{3}{*}{3} & \multirow{3}{*}{ SM } & Susceptible & 120 & 13 & 133 & \multirow{3}{*}{0.8} \\
\hline & & Resistant & 2 & 86 & 88 & \\
\hline & & Total & 122 & 99 & 221 & \\
\hline \multirow{3}{*}{4} & \multirow{3}{*}{ EMB } & Susceptible & 162 & 11 & 173 & \multirow{3}{*}{0.84} \\
\hline & & Resistant & 2 & 46 & 48 & \\
\hline & & Total & 164 & 57 & 221 & \\
\hline
\end{tabular}

\section{Discussion}

During this study, a total of 862 sputum samples were collected from the patients attending NTC. The numbers of male patients were $588(68.2 \%)$ and females were 274 $(31.8 \%)$. Out of 862 samples, $226(26.2 \%)$ samples were smear-positive for AFB. The highest numbers of AFB positive cases were seen in male patients than in female. This might be due to more exposure to external environment than females for their job and other activities, and also infected women may progress more frequently to disease and die more rapidly, leaving a cohort with a low prevalence of infection. All 226 smear positive samples and 97 smear negative samples were processed for culture. Out of these total, 221 (68.4\%) showed significant growth, $92(28.5 \%)$ samples were culture negative and $10(3.1 \%)$ were contaminated.

In the treatment and control of infectious disease caused by pathogen, susceptibility test is used to select effective antimicrobial drugs. Susceptibility test is also performed to determine the changing pattern of susceptibility among pathogens to antimicrobial drugs. Since drug-resistant TB has increased in incidence and interfered with TB control programs, monitoring of drug resistance patterns is very much important to prevent MBR-TB outbreaks. So, all isolates of $M$. tuberculosis should be tested for their susceptibilities to the primary anti-tubercular drugs.

Of the conventional culture-based techniques for antimicrobial susceptibility testing, the Resistance Ratio (RR) and the Proportion (PR) methods are commonly used. The resistance ratio method is still in use in many countries especially the United Kingdom ${ }^{10}$. However, WHO has recommended the use of the proportion method to be used for determining drug susceptibility of $M$. tuberculosis.

To determine the correlation of the RR and the PR methods for susceptibility testing of $M$. tuberculosis to the four primary anti-tubercular drugs, all 221 biochemically confirmed isolates were enrolled in this study. In general, the percentages of agreement determined by both methods were high with regard to all drugs tested. This finding was concordant with similar studies done by Laszlo ${ }^{11}$ which gave overall agreement of both methods higher than $95 \%$ to all drugs tested. Similarly Snider ${ }^{12}$ in a large scale comparative study of drug susceptibility testing of $M$. tuberculosis stated that a level of agreement of 90 to $95 \%$ between two tests must be considered good. This criterion reveals the good agreement rate between both methods in this study.

Both methods vary greatly in drug concentrations and interpretation of the drug resistance results. Since this study was 
performed by using the same inoculum size of each isolate adjusted to McFarland No. 1 for testing by both methods at the same time, no variation in inoculum size occurred. The rate of at least one or more primary drug resistance by the RR method, 125 (56.6\%) was slightly less than that of the PR method, $127(57.5 \%)$. For the single drug resistance determined by both methods, distribution rate of resistance to all drugs had no difference. The rate of two and three drugs resistance were also almost similar by both methods. But the rate of four drugs resistance in this study was slightly different between these methods. Siddiqi13 showed the variations of the results have always been a problem for in vitro susceptibility testing especially at lower concentrations. Two concentrations of all drugs were used by both methods, and the high concentration had the percentage of resistance less than low concentration (result not shown). The rate of MDR-TB in this study was similar between these methods. It was $64(29 \%)$ by RR method and 66 $(29.9 \%)$ by PR method. The resistance rate was higher because most of the isolates were from relapse, after defaulted, treatment failure and chronic cases (case type not shown in the result section).

There may be several important factors of different susceptibility results: variation in drug stability, and preparation of inoculum size. Susceptibility test results not only depend on the presence or absence of growth on the control and drug-containing media, the inoculum for each culture must also be carefully controlled. ${ }^{15}$ Furthermore, antimicrobial susceptibility test should be performed preferably with an inexpensive and relatively simple technique.

The RR method compares the resistance of the unknown strain with that of the control strain on the same batch of medium. In this study, $\mathrm{H}_{37} \mathrm{Rv}$ strain was used as control. Smooth suspensions must be used. Large clumps of bacilli give irregular results and make reading difficult. Resistance can be expressed as the ratio of the MIC (Minimum Inhibitory Concentration) of the test strain to the MIC of the control strain in the same test. The resistant strains give the ratio of 4 or more. ${ }^{16}$ To determine the ratio, too many sets of media containing two fold dilutions of the drug should be prepared which is very tedious and expensive. So, in this study for each drug tested, only two concentrations were used for both test and control strain. The RR method was convenient for inoculum preparation and required a shorter time. Interpretation of the result was rather simple.

For PR method, several dilutions of the inoculum were made and both drug-free and drugcontaining media were inoculated. This method was technically very difficult. There was much risks attached to standardizing the inocula than with the RR method. However, there are several new methods e.g. E-test, Alamar blue assay, DNA probes and molecular finger-printing, but these methods are more expensive, require specialized equipment and highly skilled personnel. Thus, they are difficult for use in general laboratories although they provide results within 1-5 days.

While comparing the RR and the PR methods, Mc Nemar $\chi^{2}$ test showed no significant difference between both methods and there were very good agreement rates of the both methods when compared using kappa analysis with kappa value $0.864,0.854,0.861$, and 0.838 for $\mathrm{INH}$, RFP, SM and EMB respectively. Similar results were shown by the study done by Tansuphasiri ${ }^{8}$ with kappa value $0.929,0.621,0.893$ and 0.620 for INH, RFP, SM and EMB respectively. The closer kappa is to 1.0, the higher the accuracy of the data.

Among the tested antimicrobial agents, this in vitro testing showed EMB was the most effective drug followed by RFP. EMB is effective against drug resistant strains of $M$. tuberculosis, with bacteriostatic effect. Similarly, RFP is active against both drug sensitive and resistant strains of $M$. tuberculosis. Literature reviews and the present study clearly showed that both RFP and EMB are most effective drugs. Higher rates of resistance to $\mathrm{INH}$ and SM might be due to the fact that because of their low cost and wide spread use in the treatment of TB. ${ }^{17}$

\section{Conclusion}

In this study, the highest agreement has been observed between the resistance ratio and 
proportion methods (with agreement rate to $\mathrm{INH}$, RFP, SM and EMB of 93.21, 93.67, 93.21, and $94.12 \%$ respectively) and the correlation between both methods to the four primary anti-tubercular drugs tested was not statistically significantly different by Mc Nemar $\chi^{2}$ test ( $p>0.05$ ). Similarly, the kappa (k) value for INH, RFP, SM and EMB were $0.864,0.854,0.861$ and 0.838 respectively which showed good agreement between both methods.

The proportion method has been recommended by WHO for determining drug susceptibility of $M$. tuberculosis however, the resistance ratio method is also equally compatible and hence can be used for drug susceptibility testing. The proper determination of drug resistance by the proper method is helpful to minimize the spread of drugresistant TB.

\section{Acknowledgements}

The authors would like to thank all the staffs of National Tuberculosis Centre and SAARC Tuberculosis and HIVIAIDS Centre, Thimi, Bhaktapur, Nepal for their constant help during this study.

\section{References}

1) World Health Organization. Tuberculosis facts. WHO, Geneva 2007; WHO / HTM / STB / fact sheet / 2007.1

2) Annual Report, National Tuberculosis Control Programme, 2005/2006. Thimi, Bhaktapur, Nepal: National Tuberculosis Centre, 2007

3) World Health Organization. Treatment of Tuberculosis, $3^{\text {rd }}$ edition, WHO, Geneva 2003; WHO/CDS/TB/2003.313:11

4) Rieder HL, Chonde TM, Myking $\mathrm{H}$, et al - $A$. The public health service national tuberculosis reference laboratory and the national laboratory network: Minimum requirements, role and operation in a low-income country. IUATLD, France, 1998

5) World Health Organization. Laboratory services in tuberculosis control Part II and III. WHO, Geneva 1998; WHO/TB/98.258

6) Fujiki A. TB Bacteriology examination to Stop TB. The Research Institute of Tuberculosis, JICA, Japan, 2001
7) Tuberculosis Program World Health Organization/International Union Against Tuberculosis and Lung Disease. Guidelines for surveillance of drug resistance tuberculosis. Document WHO/TB/94

8) Tansuphasiri U, Subpaiboon $S$ and Rienthong $S$. Comparison of the resistance ratio and proportion methods for antimicrobial susceptibility testing of Mycobacterium tuberculosis. J Med Assoc Thai. 2001;84:1467-1476

9) Thompson WD and Walter SD. Kappa and the concept of independent errors. J Clin Epidem. 1988;41:969-970

10) Kent PT and Kubica GP. Public health Mycobacteriology: A guide for the level III laboratory. US Department of Health and Human services, Public health service, CDC, Georgia, 1985

11) Laszlo A, Gill P, Handzel V, et al - Conventional and radiometric drug susceptibility testing of Mycobacterium tuberculosis complex. J Clin Microbiol. 1983;18(6):1335-1339

12) Snider DEJ, Good RG, Kilburn JO, Laskowski LFJ, Lusk RH, Marr JJ, Reggiardo $Z$ and Middlebrook $G$. Rapid susceptibility testing of Mycobacterium tuberculosis. Am Rev Respir Dis. 1981:123:402-406

13) Siddiqi SH, Hawkins JE and Laszlo $A$. Interlaboratory drug susceptibility testing of Mycobacterium tuberculosis by a radiometric procedure and two conventional methods. J Clin Microbiol. 1985;22:919-923

14) Rist N. Nature and development of resistance of tubercle bacilli to chemotherapeutic agents In: Barry CV (ed.) Chemotherapy of tuberculosis. London, Butterworths: 1964:192-227

15) Canetti G, Fox W, Khomenko A, et al - Advances in techniques of testing mycobacterial drug sensitivity, and the use of sensitivity tests in tuberculosis control programmes. Bull World Health Organ. 1969;41:21-43

16) Grange JM. Mycobacterium In: Greenwood $D$, Slack RCB and Peuthere JF (eds.) Medical microbiology $A$ guide to microbial infections: pathogenesis, immunity, laboratory diagnosis and control. $16^{\text {th }}$ edition, Churchill Livingstone, India, 2006;200-209

17) Rijal B, Rahman MM and Bam DS. Multidrug resistant tuberculosis: an overview of the SAARC region. STC newsletter 2002;12(1):13-14

18) Sharma $P K$ and Chaudhary A K. Statistical methods. 1st edition, Khanal Books Prakashan, Kathmandu, 2002; 229-404 